\title{
High-dose antiviral therapy does not prevent short episodes of HSV-2 reactivation
}

The prevalence of herpes simplex virus type 2 (HSV-2) remains a global concern despite the increasing use of antiviral medications such as aciclovir and valaciclovir. Although these drugs are associated with symptomatic improvement, they do not completely eliminate the risk of sexual transmission. HSV-2 shedding (when latent virus in neurons becomes active and travels to the site of infection) has recently been reported to occur three times more frequently than previously thought, in episodes that are $<24 \mathrm{~h}$ in duration. Current standard-dose antiviral therapy is effective at suppressing long periods of reactivation but can it abrogate these shorter episodes?

Christine Johnston and colleagues at the University of Washington in Seattle set out to answer this question, and their results were published recently in the Lancet. "We hypothesized that short episodes of genital HSV-2 shedding may account for residual risk transmission during suppressive therapy. We also hypothesized that higher doses of aciclovir and valaciclovir would better suppress this breakthrough viral shedding," explains Johnston.

Researchers performed three separate open-label cross-over trials comparing various doses of aciclovir and valaciclovir in a total of 90 HSV-2-seropositive adults. Patients received the study medication for a prespecified time (4-7 weeks), then had a 1-week break before crossing over to the comparative drug schedule. Genital swabs were self-collected by patients four times a day and evaluated for HSV-2 on Western

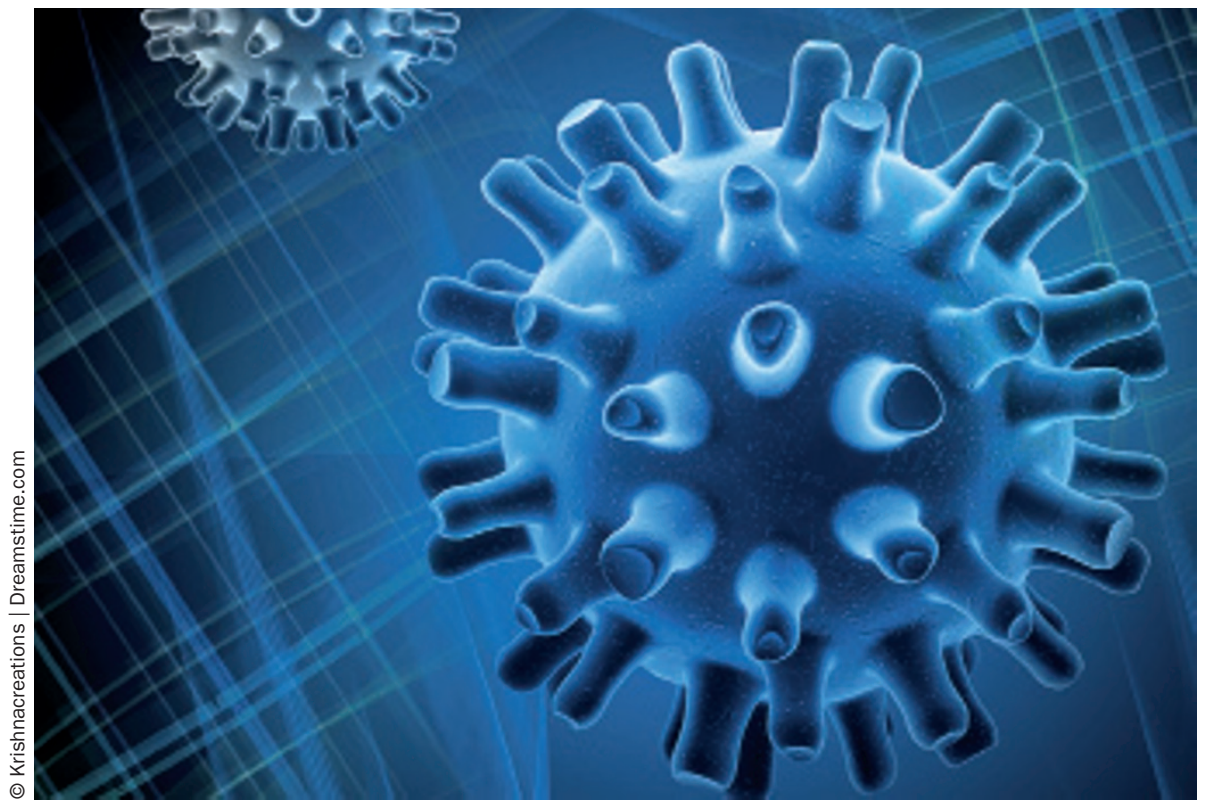

blot and PCR. Symptoms or lesions were recorded on a daily basis.

Consistent with a dose-response effect, lower rates of genital HSV-2 shedding were observed in patients who received high-dose aciclovir $(800 \mathrm{mg}$ three times daily) or high-dose valaciclovir (1 $\mathrm{g}$ three times daily) than in those who received no medication, standard-dose aciclovir (400 mg twice daily) or standard-dose valaciclovir (500 mg daily). However, breakthrough reactivation occurred frequently at all drug doses, even in patients on high-dose valaciclovir. Episode frequency did not differ between standarddose valaciclovir and high-dose aciclovir $(P=0.54)$ or between standard-dose and high-dose valaciclovir $(P=0.34)$.
These data suggest that even highdose antiviral therapy is insufficient. "Our findings underscore the need for new approaches to control the HSV-2 epidemic, including new antiviral and immunotherapeutic agents. Continued investigation into the prevention of genital $\mathrm{HSV}$ infection is also crucial. Ultimately, we hope that further research will lead to an effective prophylactic HSV vaccine," says Johnston.

Sarah Payton

Original article Johnston, C. et al. Standard-dose and high-
dose daily antiviral therapy for short episodes of genital
HSV-2 reactivation: three randomised, open-label, cross-
over trials. Lancet $379,641-647$ (2012)

